\section{SUPPRESSION OF NICKEL OUT-DIFFUSION FROM POROUS NICKEL-TITANIUM SHAPE MEMORY ALLOY BY PLASMA IMMERSION ION IMPLANTATION}

\author{
Joan P. Y. Ho, S. L. Wu, Ray W. Y. Poon, X. Y. Liu, C. Y. Chu \\ ng, Paul K. Chu* \\ Department of Physics \& Materials Scien \\ City University of Hong Kong \\ Tat Chee Avenue, Kowloon, Hong Kong
}

Kelvin W. K. Yeung, William W. Lu, Kenneth M. C.Cheung

Department of Orthopaedics and Traumatology

The University of Hong Kong

Pokfulam, Hong Kong

Porous Nickel Titanium is a promising material for medical application not only because of its super elasticity and shape memory effect but also the porous structure which may enhance bone growth due to the increased surface area. It is thus especially suitable for bone tissue in-growth and fixation of biomedical implants. However, like its dense counterpart, $\mathrm{Ni}$ leaching from the materials causes health concern. Thus, in order to suppress $\mathrm{Ni}$ diffusion from the materials to body fluids and tissues in humans, a diffusion barrier or similar structure must be introduced. In this work, we produced this diffusion barrier layer by oxygen or nitrogen plasma immersion ion implantation (PIII). In vitro tests were conducted by immersing the plasma-treated $\mathrm{NiTi}$ into simulated body fluid (SBF) at $37 \pm 0.5^{\circ} \mathrm{C}$ for 5 weeks and the resulting SBF was analyzed for $\mathrm{Ni}$ and $\mathrm{Ti}$ using inductively-co upled plasma mass spectrometry (ICMPS). Our results show $t$ hat Ni leaching is significantly mitigated by both nitrogen and oxygen PIII.

\footnotetext{
* Corresponding author: paul.chu@cityu.edu.hk

** Work supported by Hong Kong Research Grants Council (RGC) Central Allocation Scheme CityU 1/04C.
}

\author{
MICROWAVE PLASMA TORCH ABATEMENT OF \\ $\mathrm{NF}_{3}$ AND SF $_{6}$ \\ Yong C. Hong and Han S. Uhm \\ Department of Molecular Science and Technology, Ajou \\ University, Suwon 443-749, Korea \\ Byung J. Chun \\ LWSmartech Co., Ltd., Kyonggido Small Business Center, \\ Suwon 443-766, Korea \\ Sun K. Lee and Sang K. Hwang \\ M.A.T. Co., Ltd., 50-2 Mangjung-Ri, Gongdo-Eup, Ansung \\ 456-823, Korea
}

Fluorinated compounds (FCs) are essential to the semiconductor manufacturing process for plasma chamber cleaning and plasma etching. Because FCs have extremely long atmospheric lifetimes and are strong infrared absorbers, efforts have been undertaken to identify methods to reduce atmospheric emissions. Many methods for FC abatement have been suggested, such as wet and burning scrubber, and lowpressure plasma device. In this work, an atmospheric pressure microwave plasma torch as a new method for PFC abatement was presented. Detailed experiments were conducted on abatement of $\mathrm{NF}_{3}$ and $\mathrm{SF}_{6}$ in terms of destruction and removal efficiency (DRE) using Fourier Transform Infrared (FTIR).

Swirl gas, compressed air, for stable plasma was injected with main mixture of $\mathrm{N}_{2}, \mathrm{NF}_{3}$ or $\mathrm{SF}_{6}$, and $\mathrm{C}_{2} \mathrm{H}_{4}$ as an additive gas into the microwave plasma torch. Destruction and removal efficiencies of up to $99.1 \%$ for $\mathrm{NF}_{3}$ were achieved without an additive gas by applying microwave powers from 0.8 to $1.2 \mathrm{~kW}$. Also, DRE determined from FTIR data for $\mathrm{SF}_{6}$ was obtained $90.1 \%$ using applied microwave power of 1.4 $\mathrm{kW}$. Experimental results indicate that the microwave plasma abatement device for PFC destruction can successfully eliminate FCs in the semiconductor industry. 